\title{
Phenolic Compounds and Antioxidant Activity of Juices from Ten Iranian Pomegranate Cultivars Depend on Extraction
}

\author{
Hamidreza Akhavan, ${ }^{1}$ Mohsen Barzegar, ${ }^{2}$ Helmut Weidlich, ${ }^{3}$ and Benno F. Zimmermann ${ }^{3,4}$ \\ ${ }^{1}$ Department of Food Science and Technology, Faculty of Agriculture, Shahid Bahonar University of Kerman, \\ P.O. Box 76169-133, Kerman, Iran \\ ${ }^{2}$ Department of Food Science and Technology, Faculty of Agriculture, Tarbiat Modares University, \\ P.O. Box 14115-336, Tehran, Iran \\ ${ }^{3}$ Institut Prof. Dr. Georg Kurz GmbH, Stöckheimer Weg 1, 50829 Köln, Germany \\ ${ }^{4}$ Department of Nutritional and Food Sciences, University of Bonn, Römerstraße 164, 53117 Bonn, Germany \\ Correspondence should be addressed to Benno F. Zimmermann; benno.zimmermann@uni-bonn.de
}

Received 17 September 2015; Revised 5 November 2015; Accepted 8 November 2015

Academic Editor: Patricia Valentao

Copyright @ 2015 Hamidreza Akhavan et al. This is an open access article distributed under the Creative Commons Attribution License, which permits unrestricted use, distribution, and reproduction in any medium, provided the original work is properly cited.

Phenolic compounds and antioxidant activities of ten juices from arils and whole pomegranate cultivars grown in Iran were studied. Phenolic contents and antioxidant activities of juices from whole pomegranate fruit were significantly higher than juices from pomegranate arils, but the variety has a greater influence than the processing method. The main phenolics in the studied juices were punicalagin A $(5.40-285 \mathrm{mg} / \mathrm{L})$, punicalagin B $(25.9-884 \mathrm{mg} / \mathrm{L})$, and ellagic acid $(17.4-928 \mathrm{mg} / \mathrm{L})$. The major and minor anthocyanins of cyanidin 3,5-diglucoside $(0.7-94.7 \mathrm{mg} / \mathrm{L})$, followed by cyanidin 3-glucoside $(0.5-52.5 \mathrm{mg} / \mathrm{L})$, pelargonidin 3,5-diglucoside + delphinidin 3-glucoside (0-10.3 mg/L), delphinidin 3,5-diglucoside $(0-7.68 \mathrm{mg} / \mathrm{L})$, pelargonidin 3-glucoside $(0$ $9.40 \mathrm{mg} / \mathrm{L})$, and cyanidin-pentoside $(0-1.13 \mathrm{mg} / \mathrm{L})$ were identified; the latter anthocyanin as well as cyanidin-pentoside-hexoside and delphinidin-pentoside were detected for the first time in Iranian pomegranates. The total phenolic contents were in the range of $220-2931 \mathrm{mg} / 100 \mathrm{~mL}$. The results indicate that the pomegranate phenolics are not only influenced by extraction method but alsoand even more-affected by the cultivar. Moreover, a good correlation was observed between total phenolic content and ABTS and FRAP methods in all pomegranate juices $(>0.90)$. The results of current research can help to select the pomegranate cultivars for commercial juice production.

\section{Introduction}

Pomegranate fruit (Punica granatum L.) and its products are rich sources of bioactive compounds such as flavonoids, ellagitannins, mainly punicalagins, ellagic acid, and punicalins that also affect visual appearance and flavour of pomegranate juices $[1,2]$. These compounds are discussed as health promoting, but excessive amounts of polyphenols can form undesirable astringent taste. This fruit is one of the most important commercial fruits in Iran and its total production in 2013 was $\sim 910,000$ tons [3]. The pomegranate is consumed worldwide as fresh fruit, juice, jam, and jelly, and nutritional supplements.
In recent years, the physicochemical properties of pomegranate fruit such as total phenolic, total anthocyanin, and total tannin contents and antioxidant activity assay with different methods $[1,2,4-11]$ and individual phenolic compounds such as flavonoids (including anthocyanins), ellagitannins, mainly punicalagins, ellagic acid, and punicalins $[1,2,4,5,11-14]$ have been published in literature.

Thus, the major phenolic compounds of pomegranate juices have been documented, but no information is available about identification and quantification of phenolic compounds and antioxidant activities of juices obtained from arils only and from whole Iranian pomegranate cultivars. This study can help to select pomegranate cultivars for commercial 
use. Therefore, the aims of this study were (1) to quantify individual phenolic compounds, (2) to determine total phenolic content as well as antioxidant activity, and (3) to study correlation of these parameters in pomegranate juices obtained from arils and whole pomegranates of different cultivars.

\section{Materials and Methods}

2.1. Plant Material. The following ten pomegranate cultivars were harvested in the last week of September 2011 from mature trees (16 years old) growing in the Agricultural Research Center of Yazd province, Iran $\left(31^{\circ} 54^{\prime} 54^{\prime \prime} \mathrm{N}\right.$, $\left.54^{\circ} 16^{\prime} 37^{\prime \prime} \mathrm{E}\right)$ : Vahshe Kane Tehran (VKT), Gorche Shahvar Yazdi (GSY), Malase Yazdi (MY), Mesri Torshe Kazeron (MTK), Jangali Pust Germeze Rodbare Torsh (JPGRT), Torshe Mamoli Lasjer (TML), Ardestani Torshe Semnan (ATS), Sefeede Robi Aval Brojen (SRAB), Pust Syahe Yazd (PSY), and Malase Porbarij Stahban (MPS).

The trees were grown under the same environmental conditions, basin irrigation ( 2-3 times per month during growing season from March to September 2011), and routine cultural practices suitable for commercial fruit production. The trees were planted with an average of $6 \mathrm{~m}$ within rows and $3 \mathrm{~m}$ between them. Yazd province is located in a desert environment with an annual precipitation of $<100 \mathrm{~mm}$. The average temperature, the amount of rainfall, and relative humidity in growing season of 2011 were $25.0^{\circ} \mathrm{C}, 14.0 \mathrm{~mm}$, and $23.6 \%$, respectively. The soil texture is sandy-loam, with EC $=4.12 \mathrm{dSm}^{-1}$ and soil $\mathrm{pH}=7.21$. Ten fruits (approximately $2.5 \mathrm{~kg}$ ) from four trees (2-3 fruits from each tree) were harvested when fully matured according to commercial practice. It should be noted that the pomegranate fruits in Yazd province were fully matured and harvested between 15 th and 30th September [12].

2.2. Chemicals. Punicalagin and cyanidin 3-O-glucoside standards were purchased from Phytolab (Vestenbergsgreuth, Germany) and ellagic acid was purchased from Dr. Jacob (Taunusstein, Germany). The Folin-Ciocalteu's phenol reagent (Merck, Germany), ABTS [2,20-azinobis(3ethylbenzothiazoline-6-sulfonic acid)], DPPH radical (1,1diphenyl-2-picrylhydrazyl), and TPTZ (2,4,6-tripyridyl-striazine) were purchased from Sigma-Aldrich, USA. All solvents used as UHPLC eluents were LC-MS grade; optigrade water was obtained from Promochem (Wesel, Germany), acetonitrile from Mallinckrodt Baker (Deventer, The Netherlands), and formic acid from Fluka/Sigma Aldrich.

2.3. Preparation of Pomegranate Juice. Each pomegranate cultivar was washed in cold tap water and drained. The fruits were pressed using a self-made press consisting of a movable car lifter and a stainless steel pot creating a pressure of $67.4 \mathrm{kp} / \mathrm{cm}^{2}$. The supernatant juice was decanted and frozen at $-20^{\circ} \mathrm{C}$ until analysis. Juices were filtered through disposable membrane filters (regenerated cellulose, $0.2 \mu \mathrm{m}$ pore size, Macherey + Nagel, Düren, Germany) and analysed without any further sample pretreatment. Suffixes
A and W (such as MYA and MYW) at each abbreviation of pomegranate cultivars indicate the juices obtained from arils (i.e., without the exocarp and the endocarp) and whole pomegranate juices, respectively. Approximately $2.5 \mathrm{~kg}$ of pomegranate fruits was cut in two halves, in order to obtain aril juice and whole pomegranate juice from the same fruits. For the aril juices, the arils were separated manually and the inner white parts of the fruit were removed.

2.4. UHPLC Analysis, Identification, and Quantification of Phenolic Compounds. For analysis of phenolic compounds, an Acquity UPLC-UV-MS system (Waters, Milford, MA, USA) was used as described by Feuereisen et al. [15].

Anthocyanins were separated using an Acquity HSS-T3 RP18 column $(100 \mathrm{~mm} \times 2.1 \mathrm{~mm}, 1.8 \mu \mathrm{m}$ particle size $)$ from Waters with a guard column $(5 \mathrm{~mm} \times 2.1 \mathrm{~mm})$. Eluent A was water $/ 1 \%$ formic acid; eluent B was acetonitrile $/ 0.1 \%$ formic acid. The gradient program at a flow rate of $0.45 \mathrm{~mL} / \mathrm{min}$ was as follows: $0-0.2 \mathrm{~min}, 0 \% \mathrm{~B} ; 0.6 \mathrm{~min}, 5 \% \mathrm{~B} ; 20 \mathrm{~min}$, $12 \% \mathrm{~B}$; $21-24 \mathrm{~min}, 95 \% \mathrm{~B}$; and $25-27 \mathrm{~min}, 0 \% \mathrm{~B}$. The mass spectrometer was tuned using a solution of cyanidin 3-Oglucoside. The following parameters were obtained: capillary voltage $1.6 \mathrm{kV}$, cone voltage $32 \mathrm{~V}$, extractor voltage $3.0 \mathrm{~V}$, RF voltage $1.3 \mathrm{~V}$, source temperature $150^{\circ} \mathrm{C}$, desolvation temperature $450^{\circ} \mathrm{C}$, cone gas (nitrogen) flow $50 \mathrm{~L} / \mathrm{h}$, and desolvation gas (nitrogen) flow $800 \mathrm{~L} / \mathrm{h}$. The collision gas (argon) flow used in tandem mass spectrometry experiments was $0.3 \mathrm{~mL} / \mathrm{min}$. MS detection was used for confirmation of peak identity in selected reaction monitoring (SRM) mode. For quantification, an external calibration of cyanidin 3-Oglucoside was used (detection wavelength of $500 \mathrm{~nm}$ ). Each sample was analyzed once. All compounds were identified by MS (SRM) and quantified by UV-Vis.

For the separation of punicalagin and ellagic acid, an Acquity BEH Phenyl column $(50 \mathrm{~mm} \times 2.1 \mathrm{~mm}$, particle size $1.7 \mu \mathrm{m})$ from Waters with a guard column $(5 \mathrm{~mm} \times 2.1 \mathrm{~mm})$ was used. Eluent A was water $/ 0.1 \%$ formic acid; eluent B was acetonitrile $/ 0.1 \%$ formic acid. The gradient program at a flow rate of $0.5 \mathrm{~mL} / \mathrm{min}$ was as follows: $0-3 \mathrm{~min}, 0 \% \mathrm{~B} ; 13 \mathrm{~min}, 35 \%$ B; $13.2 \mathrm{~min}, 95 \% \mathrm{~B}$; $13.2-14 \mathrm{~min}, 95 \% \mathrm{~B}$; and $14.2-15 \mathrm{~min}, 0 \%$ B. Each sample was analysed in duplicate.

The mass spectrometer was tuned using a solution of punicalagin, resulting in the following parameters: capillary voltage $-2.0 \mathrm{kV}$, cone voltage $34 \mathrm{~V}$, extractor voltage $2.0 \mathrm{~V}$, RF voltage $0.3 \mathrm{~V}$, source temperature $150^{\circ} \mathrm{C}$, desolvation temperature $450^{\circ} \mathrm{C}$, cone gas (nitrogen) flow $50 \mathrm{~L} / \mathrm{h}$, and desolvation gas (nitrogen) flow $900 \mathrm{~L} / \mathrm{h}$. The collision gas (argon) flow used in tandem mass spectrometry experiments was $0.3 \mathrm{~mL} / \mathrm{min}$. For quantification, an external calibration of punicalagin and ellagic acid was used (detection wavelength of $360 \mathrm{~nm}$ ). All compounds were identified by MS (SRM) and quantified by UV.

2.5. Determination of Total Phenolic Content (TPC) and Antioxidant Activity of Juices. Total phenolic content was measured using Folin-Ciocalteu method described by Tezcan et al. [8] using gallic acid as reference. The antioxidant capacity of pomegranate juices was evaluated based on free 
TABLE 1: Contents of individual phenolic compounds (mg/L) in pomegranate juices (PJ).

\begin{tabular}{|c|c|c|c|c|c|c|c|}
\hline PJ from arils & Punicalagin A & Punicalagin B & Ellagic acid & PJ from whole fruits & Punicalagin A & Punicalagin B & Ellagic acid \\
\hline VKTA & $15.9 \pm 0.1^{\mathrm{ef} *}$ & $36.7 \pm 2.6^{\mathrm{gh}}$ & $74.8 \pm 0.6^{\mathrm{g}}$ & VKTW & $66.5 \pm 0.6^{\mathrm{e}}$ & $219.6 \pm 0.6^{\mathrm{d}}$ & $245.9 \pm 4.9^{\mathrm{d}}$ \\
\hline GSYA & $13.2 \pm 0.1^{\mathrm{f}}$ & $48.5 \pm 0.3^{g}$ & $108.1 \pm 3.3^{f}$ & GSYW & $285.0 \pm 2.8^{\mathrm{a}}$ & $884.3 \pm 52.9^{\mathrm{a}}$ & $928.3 \pm 20.2^{\mathrm{a}}$ \\
\hline MYA & $73.1 \pm 1.4^{\mathrm{b}}$ & $258.9 \pm 4.6^{\mathrm{b}}$ & $115.5 \pm 0.7^{\mathrm{de}}$ & MYW & $74.6 \pm 0.5^{\mathrm{e}}$ & $263.1 \pm 5.1^{\mathrm{d}}$ & $121.2 \pm 0.1^{\mathrm{f}}$ \\
\hline MTKA & $69.8 \pm 1.8^{\mathrm{b}}$ & $240.7 \pm 2.6^{c}$ & $67.1 \pm 2.1^{\mathrm{h}}$ & MTKW & $134.6 \pm 1.9^{c}$ & $483.8 \pm 6.1^{\mathrm{c}}$ & $138.5 \pm 2.1^{\mathrm{f}}$ \\
\hline JPGRTA & $129.0 \pm 2.4^{\mathrm{a}}$ & $478.4 \pm 6.3^{\mathrm{a}}$ & $132.6 \pm 1.4^{\mathrm{b}}$ & JPGRTW & $175.1 \pm 6.0^{\mathrm{b}}$ & $651.9 \pm 19.9^{\mathrm{b}}$ & $177.6 \pm 4.0^{\mathrm{e}}$ \\
\hline TMLA & $19.4 \pm 0.7^{\mathrm{e}}$ & $48.4 \pm 0.9^{g}$ & $120.1 \pm 1.6^{\mathrm{cd}}$ & TMLW & $119.2 \pm 3.0^{\mathrm{d}}$ & $449.6 \pm 5.7^{\mathrm{c}}$ & $339.9 \pm 0.1^{\mathrm{c}}$ \\
\hline ATSA & $36.5 \pm 1.6^{\mathrm{d}}$ & $101.3 \pm 3.8^{\mathrm{f}}$ & $155.9 \pm 2.6^{\mathrm{a}}$ & ATSW & $182.5 \pm 2.4^{\mathrm{b}}$ & $608.5 \pm 9.5^{\mathrm{b}}$ & $460.5 \pm 4.1^{\mathrm{b}}$ \\
\hline SRABA & $54.3 \pm 0.5^{\mathrm{c}}$ & $190.3 \pm 2.9^{\mathrm{d}}$ & $110.7 \pm 0.4^{\mathrm{ef}}$ & SRABW & $115.3 \pm 3.1^{\mathrm{d}}$ & $434.1 \pm 0.1^{\mathrm{c}}$ & $315.4 \pm 2.6^{c}$ \\
\hline PSYA & $5.4 \pm 0.0^{\mathrm{g}}$ & $25.9 \pm 1.1^{\mathrm{h}}$ & $17.4 \pm 1.9^{\mathrm{i}}$ & PSYW & $23.3 \pm 1.0^{\mathrm{f}}$ & $132.8 \pm 3.6^{\mathrm{e}}$ & $267.3 \pm 1.0^{\mathrm{d}}$ \\
\hline MPSA & $50.8 \pm 0.9^{c}$ & $149.2 \pm 3.0^{\mathrm{e}}$ & $123.3 \pm 1.6^{\mathrm{c}}$ & MPSW & $180.9 \pm 3.9^{\mathrm{b}}$ & $650.1 \pm 1.3^{\mathrm{b}}$ & $261.8 \pm 2.6^{\mathrm{d}}$ \\
\hline
\end{tabular}

${ }^{*}$ Different letters in the same column present significant difference at $p<0.05$.

radical scavenging capacity by DPPH radical (expressed as inhibition percentage) and ABTS radical (expressed as mg Lascorbic acid/100 mL juice) and with iron-reducing capacity by FRAP method (expressed in $\mathrm{mmol} \mathrm{Fe}^{2+} / 100 \mathrm{~mL}$ juice) $[7,8]$.

2.6. Statistical Analysis. The data were subjected to analyses of variance (ANOVA) using SPSS 17.0. For the comparison of significance between cultivars, Duncan's multiple range test $(P<0.05)$ was used as post hoc test. Results are the means \pm SD of two determinations for each juice.

\section{Results and Discussion}

3.1. Punicalagins and Ellagic Acids. In this study, the major individual phenolic compounds of ten pomegranate cultivars were identified by HPLC/PDA/MS ${ }^{2}$, namely, punicalagin A, punicalagin $B$, and ellagic acid (Table 1), besides anthocyanins (see the next paragraph). These results are in accordance with previously reports on other pomegranate cultivars $[1,2,4,11$, 16].

Gallic acid, gallagic acid, and galloyl glucose were found in minor amounts only and therefore not considered in the following evaluation. A typical chromatogram of the detected phenolic compounds of pomegranate juice (MYW cultivar) is shown in Figure 1.

The individual and total phenolic contents of all studied cultivars were significantly different $(p<0.01)$. The concentrations of the main phenolics showed wide ranges: punicalagin B $25.9-884 \mathrm{mg} / \mathrm{L}$, ellagic acid $17.4-928 \mathrm{mg} / \mathrm{L}$, and punicalagin A $5.40-285 \mathrm{mg} / \mathrm{L}$, which is in agreement with previous findings of juices from other pomegranate cultivars $[1,4,13,14]$. Pearson's correlation coefficients were high $(>0.98)$ between punicalagin A and punicalagin B at same juices (arils or whole pomegranate), but ellagic acid value was not significantly correlated with the other phenolic compounds. In each cultivar, the total individual phenolic content of whole pomegranate juices was increased by $2.5-$ $1136 \%$ compared to pomegranate aril juices. In this regard, the MY and JPGRT had increased 2.5 and 35\%, respectively, but other samples showed higher increases $(>100 \%)$. The highest increase (1136\%) was awarded to GSY cultivar.

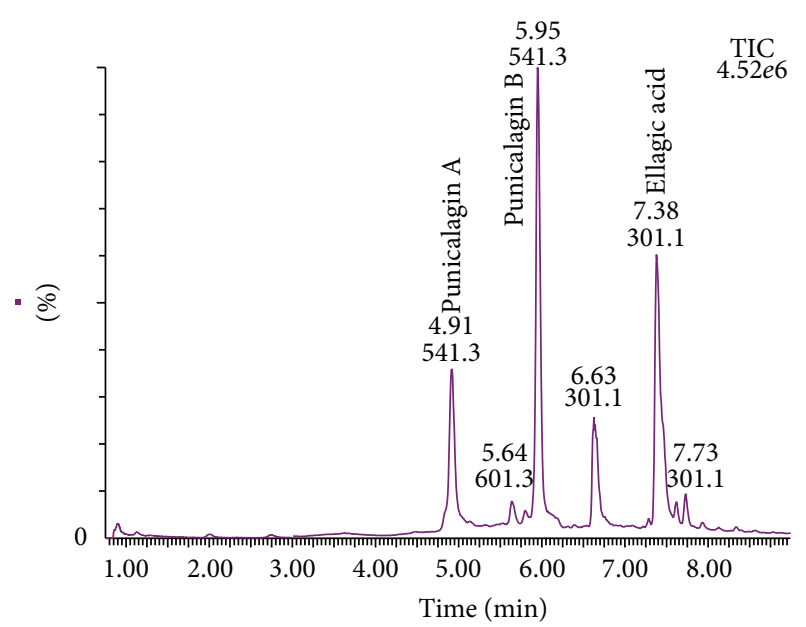

FIGURE 1: Typical chromatogram of the nonanthocyanidin phenolics in pomegranate juice (MYW) as detected by MS in selected ion monitoring (SIM) mode. Punicalagins appear as doubly charged ions. Ions with $m / z=301$ other than ellagic acid are in-source fragments of other ellagitannins.

The highest punicalagin contents among aril and whole fruit juices were found in JPGRTA (607 mg/L) and GSYW $(1169 \mathrm{mg} / \mathrm{L})$, respectively (Table 1). Punicalagin is the most characteristic ellagitannin, because it is found almost exclusively in pomegranate. However, pomegranate arils contain low or no punicalagins, but pomegranate rind parts are an abundant source. The higher punicalagin content of commercial pomegranate juices was due to the entrance of these compounds from rind parts of the fruit during processing $[1,11]$. The phenolic content of four types of "Wonderful" pomegranate juices including juices from fresh arils, frozen arils, and whole pomegranates and concentrate were evaluated [1]. Punicalagin $A$ in four types of pomegranate juices was $12.7,14.4,421$, and $435 \mathrm{mg} / \mathrm{L}$; punicalagin $\mathrm{B}$ was $10.1,11.1$, 839 , and $918 \mathrm{mg} / \mathrm{L}$, and total ellagic derivatives were 33.2, 26.5, 121 , and $264 \mathrm{mg} / \mathrm{L}$, respectively. The four major hydrolyzable tannins of punicalagin, ellagic acid, punicalin, and gallagic acid were detected in 29 different pomegranate accessions by Tzulker et al. [11]. The contents of these compounds 
significantly differed in the whole fruit homogenates. The content of punicalagin was about 10-fold higher than the punicalin and gallagic acid contents and 100-fold higher than the content of ellagic acid. Although punicalagin, punicalin, and gallagic acid were also found in aril juices (ellagic acid was below the detection level), these contents were very low in comparison to the whole fruit homogenates [11].

In literature, punicalagins content was reported to be lower in pomegranate arils than in whole pomegranate fruit or even absent in the former $[1,2,11]$. But in the current study, the cultivar JPGRT had such a high punicalagin content that the juice made from the arils of JPGRT had a higher content $(607 \mathrm{mg} / \mathrm{L})$ than the juice made from whole pomegranate of certain cultivars (MYW, TMLW, SRABW, and PSYW). Therefore, the content (or lack) of punicalagins in pomegranate juice is not only affected by extraction method, but also-and even more-by the pomegranate cultivar.

Fischer et al. [2] identified 48 compounds in 3 variants of pomegranate juices, among which 9 were anthocyanins, 2 gallotannins, 22 ellagitannins, 2 gallagyl esters, 4 hydroxybenzoic acids, and 7 hydroxycinnamic acids and 1 was dihydroflavonol. The phenolic compounds of pomegranate juice prepared from whole pomegranate at 10 bars, juice prepared from whole pomegranate at 150 bars, and juice prepared from the isolated arils at 250 bars were as follows: total gallotannins $(1.2-9.1 \mathrm{mg} / \mathrm{L})$, total ellagitannins (93.2$2074 \mathrm{mg} / \mathrm{L}$ ), total hydroxybenzoic acids (1.1-10.6 mg/L), and total hydroxycinnamic acids $(21.4-28.3 \mathrm{mg} / \mathrm{L})$. In another study, punicalagin levels of juices from the pomegranate cultivar "Mollar" ranged between 504 and $763 \mathrm{mg} / \mathrm{L}$, and punicalins were found in a lower range between 240 and $365 \mathrm{mg} / \mathrm{L}$. The free ellagic acid level was between 269 and $390 \mathrm{mg} / \mathrm{L}$ [16].

Also, Mena et al. [14] have identified 75 compounds by UHPLC-MS ${ }^{n}$, of which 21 compounds were tentatively identified for the first time in pomegranate juice of Mollar de Elche cultivar. The separated compounds included 37 ellagitannins, 6 gallotannins, 14 noncoloured flavonoids, 5 phenolic acid derivatives, 6 anthocyanins and a flavanolanthocyanin adduct, 6 lignans, and 3 organic acids.

3.2. Individual Anthocyanin Content. The individual anthocyanin contents of ten pomegranate cultivars were determined using UHPLC. A typical chromatogram of individual anthocyanins of MYA cultivar is shown in Figure 2. The applied chromatographic conditions successfully separated both the major and minor anthocyanins of pomegranate juice. The detected anthocyanins were cyanidin 3-glucoside (Cy 3G), cyanidin 3,5-diglucoside (Cy 3,5dG), delphinidin 3-glucoside (Dp 3G), delphinidin 3,5diglucoside (Dp 3,5dG), pelargonidin 3-glucoside (Pg 3G), pelargonidin 3,5-diglucoside (Pg 3,5dG), cyanidin-pentoside (Cy-pent), cyanidin-pentoside-hexoside (Cy-pent-hex), and delphinidin-pentoside (Dp-pent).

Table 2 shows the individual anthocyanin contents of the studied cultivars. The JPGRTA cultivar had the highest $(175 \mathrm{mg} / \mathrm{L})$ anthocyanin content whereas those of MPSA, ATSA, PSYA, and PSYW were lower than $5 \mathrm{mg} / \mathrm{L}$. In some cultivars, juice extraction of whole pomegranate

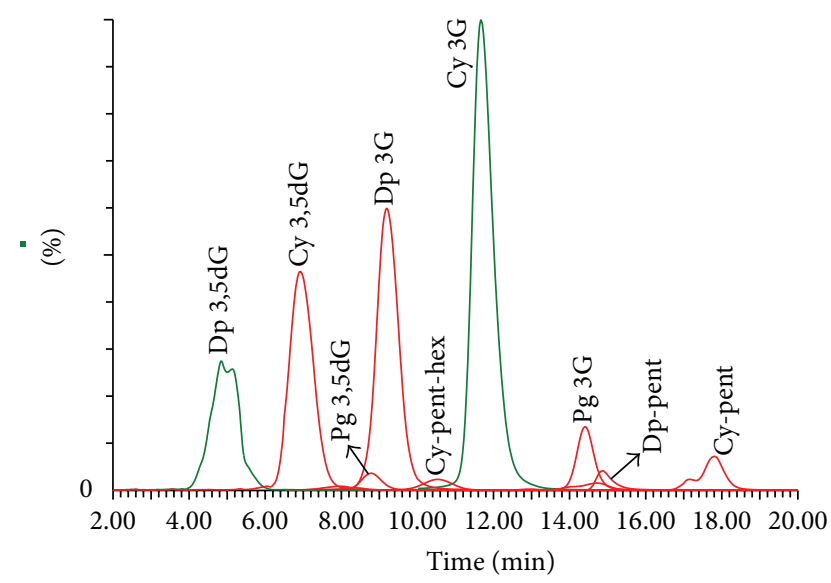

FIgURE 2: Typical chromatogram of the anthocyanidins in pomegranate juice (MYA) as detected by MS in SRM mode.

juices increased the anthocyanin content: ATSW (134\%), GSYW (47.7\%), MPSW (34.7\%), MTKW (22.3\%), and PSYW (19.6\%). In contrast, the anthocyanin contents of SRABW (21.4\%), TMLW (10.4\%), MYW (4.40\%), and JPGRTW (3.90\%) were decreased. Based on these results, it is evident that the anthocyanin content of pomegranate juices is affected by extraction methods.

According to Figure 2, nine major and minor anthocyanins were identified, namely, cyanidin 3-glucoside, cyanidin 3,5-diglucoside, delphinidin 3-glucoside, delphinidin 3,5-diglucoside, pelargonidin 3-glucoside, pelargonidin 3,5-diglucoside, cyanidin-pentoside, cyanidin-pentosidehexoside, and delphinidin-pentoside. In terms of quantity, the main anthocyanin in most of the cultivars was Cy 3,5dG $(0.7-94.7 \mathrm{mg} / \mathrm{L})$, followed by Cy $3 \mathrm{G}(0.5-52.5 \mathrm{mg} / \mathrm{L}), \mathrm{Pg}$ $3,5 \mathrm{dG}+\mathrm{Dp} 3 \mathrm{G}(0-10.3 \mathrm{mg} / \mathrm{L}), \mathrm{Dp} 3,5 \mathrm{dG}(0-9.40 \mathrm{mg} / \mathrm{L}), \mathrm{Pg}$ $3 \mathrm{G}(0-4.00 \mathrm{mg} / \mathrm{L})$, and Cy-pent $(0-1.13 \mathrm{mg} / \mathrm{L})$.

Except for Cy-pent, Dp-pent, and Cy-pent-hex, the anthocyanin profiles were consistent with previously reported researches about other pomegranate cultivars $[1,12,17-19]$. In the same pomegranate cultivars, Alighourchi et al. [12] reported the content of 6 anthocyanins in aril juices as follows: Dp 3G (2.19-16.3 mg/L), Dp 3,5dG $(2.36-63.1 \mathrm{mg} / \mathrm{L}), \quad$ Pg $3 \mathrm{G} \quad(0.26-1.36 \mathrm{mg} / \mathrm{L}), \quad$ Pg $3,5 \mathrm{dG}$ $(0.01-8.11 \mathrm{mg} / \mathrm{L})$, Cy $3 \mathrm{G}(5.78-30.4 \mathrm{mg} / \mathrm{L})$, and Cy $3,5 \mathrm{dG}$ $(4.39-166 \mathrm{mg} / \mathrm{L})$. Thus, achieving different anthocyanins content in the same pomegranate cultivars indicated the effect of agricultural and environmental conditions on the physicochemical characteristic of pomegranate fruit.

To our knowledge, Dp-pent has not been reported in pomegranate fruit so far. It is tentatively identified by the mass transition $435>303$. Fischer et al. [2] identified 9 anthocyanins of Dp 3,5dG (0.4-205 mg/L), Cy 3,5dG (95.4-271 mg/L), Pg 3,5dG (13.7-26.7 mg/L), Dp 3G (1.0$16.8 \mathrm{mg} / \mathrm{L})$, Cy $3 \mathrm{G}(9.1-27.9 \mathrm{mg} / \mathrm{L})$, Pg $3 \mathrm{G}(2.4-4.9 \mathrm{mg} / \mathrm{L})$, a cyanidin-pentoside-hexoside $(1.1-3.0 \mathrm{mg} / \mathrm{L})$, cyanidin 3rutinoside $(0.0-1.2 \mathrm{mg} / \mathrm{L})$, and a cyanidin-pentoside $(0.2-$ $0.6 \mathrm{mg} / \mathrm{L}$ ) in the three pomegranate juice variants. Gil et al. [1] reported the main pigments in the juice of the Wonderful 
TABLE 2: Contents of individual anthocyanins $(\mathrm{mg} / \mathrm{L})$ in pomegranate juices.

\begin{tabular}{|c|c|c|c|c|c|c|c|}
\hline Pomegranate juice & Dp 3,5dG & Cy $3,5 \mathrm{dG}$ & $\mathrm{Pg} 3,5 \mathrm{dG}+\mathrm{Dp} 3 \mathrm{G}$ & Cy $3 \mathrm{G}$ & $\mathrm{Pg} 3 \mathrm{G}$ & Cy-pent & Total anthocyanins \\
\hline VKTA & 0.0 & 11.7 & 0.8 & 6.6 & 0.5 & 0.0 & 19.6 \\
\hline GSYA & 0.0 & 9.1 & 1.0 & 5.5 & 0.8 & 0.0 & 16.4 \\
\hline MYA & 1.5 & 63.3 & 10.3 & 44.8 & 9.4 & 0.8 & 130.2 \\
\hline MTKA & 0.0 & 15.9 & 2.0 & 11.0 & 1.5 & 0.3 & 30.7 \\
\hline JPGRTA & 5.0 & 93.2 & 8.7 & 62.6 & 4.7 & 1.1 & 175.4 \\
\hline TMLA & 0.0 & 19.8 & 2.4 & 4.2 & 0.8 & 0.0 & 27.1 \\
\hline ATSA & 0.0 & 2.4 & 0.8 & 0.5 & 0.0 & 0.0 & 3.7 \\
\hline SRABA & 0.0 & 4.3 & 1.0 & 5.0 & 0.3 & 0.0 & 10.5 \\
\hline PSYA & 0.0 & 0.7 & 0.4 & 0.6 & 0.1 & 0.0 & 1.8 \\
\hline MPSA & 0.0 & 2.3 & 0.0 & 1.9 & 0.0 & 0.0 & 4.2 \\
\hline VKTW & 0.0 & 11.0 & 0.9 & 7.6 & 0.6 & 0.0 & 20.1 \\
\hline GSYW & 0.0 & 11.9 & 1.7 & 9.6 & 1.0 & 0.0 & 24.3 \\
\hline MYW & 1.7 & 59.8 & 9.4 & 43.8 & 9.1 & 0.7 & 124.5 \\
\hline MTKW & 0.0 & 19.7 & 2.5 & 13.3 & 1.6 & 0.4 & 37.5 \\
\hline JPGRTW & 7.7 & 94.7 & 8.7 & 52.5 & 4.0 & 1.0 & 168.5 \\
\hline TMLW & 0.0 & 17.6 & 1.9 & 4.1 & 0.7 & 0.0 & 24.3 \\
\hline ATSW & 0.0 & 6.0 & 1.2 & 1.6 & 0.0 & 0.0 & 8.8 \\
\hline SRABW & 0.0 & 3.3 & 1.0 & 3.7 & 0.3 & 0.0 & 8.3 \\
\hline PSYW & 0.0 & 0.8 & 0.5 & 0.7 & 0.2 & 0.0 & 2.1 \\
\hline MPSW & 0.0 & 3.1 & 0.0 & 2.6 & 0.0 & 0.0 & 5.7 \\
\hline
\end{tabular}

cultivar as follows: Cy 3G (59.5-128 mg/L), Dp 3G (23.695.2 mg/L), Cy 3,5dG (31.4-71.4 mg/L), Dp 3,5dG (21.1$61.1 \mathrm{mg} / \mathrm{L})$, and Pg 3G (3.9-8.5 mg/L). Mena et al. [4] reported Cy $3 \mathrm{G}(33 \%)$ as the major anthocyanin followed by Dp 3,5dG (24\%), Dp 3G (21\%), and Cy 3,5dG (19\%) and Pg 3G and Pg 3,5dG were only $3 \%$ of the total anthocyanin content. In addition, four major anthocyanins of Dp 3G, Dp 3,5dG, Cy $3 \mathrm{G}$, and Cy 3,5dG and minor amounts of Pg $3 \mathrm{G}$ and Pg 3,5dG were determined in pomegranate juices [20].

The main anthocyanin in all the cultivars was Cy 3,5dG, except for SRABA, in which Cy $3 \mathrm{G}$ was dominant. It is known that the main anthocyanin of pomegranate depends on the cultivar. The main anthocyanins were Cy 3G [1] in the American Wonderful cultivar, Cy 3G (40\%) and Cy 3,5dG (38\%) in the Spanish Mollar cultivar [19], Dp 3G in the Portuguese Assaria cultivar [17], Cy 3,5dG in 15 Iranian cultivars [12], Dp 3,5dG in 8 other Iranian cultivars [5], and Cy $3,5 \mathrm{dG}$ in a Peruvian pomegranate fruit [2].

Among different species or even cultivars of the same species, the anthocyanin content varies considerably, affected by genetic make-up, light, temperature, and agronomic factors [21]. Intrinsic properties of the product and the process such as $\mathrm{pH}$, temperature, light, oxygen, enzymes, ascorbic acids, sugars, metal ions, and copigmentation (intermolecular and intramolecular complex formations, self-association, and metal complexation) as well as chemical structure and concentration of anthocyanins can affect anthocyanin stability [22].

The variation of anthocyanin content in juices from arils and whole pomegranate can be affected by the entrance of anthocyanins from the peel into the juices. Therefore, the amount of anthocyanins of the same cultivar varied with extraction methods.

3.3. Total Phenolic Content and Antioxidant Capacity. According to the results presented in Table 3, total phenolic contents of juices from whole pomegranate fruit (942$2931 \mathrm{mg} / \mathrm{L}$ ) were significantly higher than those in arils juice (220-1267 mg/L). The GSYW cultivar had the highest $(2931 \mathrm{mg} / \mathrm{L})$ total phenolic content. In other studies, the total phenolic contents of various pomegranate cultivars showed significant differences and were 296-985 mg/100 g [9], 208$344 \mathrm{mg} / 100 \mathrm{~g}$ [7], 144-1009 mg/100 g [8], 23.7-930 mg/100 g [5], 2015-5186 mg/L [2], and 1136-3581 mg/L [16]. Different amounts of total phenolic content in various studies can be attributed to analytical methods, cultivar, maturity stage, and environmental conditions and, also, overestimation of the total individual phenolic compounds by Folin-Ciocalteu reagent due to interference with other reducing factors [2].

The antioxidant properties were measured by ABTS, FRAP, and DPPH assay (Table 3). The results showed considerable antioxidant activity of juice samples. Antioxidant activities of juices from whole pomegranate fruit were significantly higher than juices from pomegranate arils that indicated the importance of the juice extraction methods. These results are in agreement with previously reported findings $[1,11]$. The antioxidant capacity of pomegranate juice (and other fruit juices) depends on cultivar, growing region, fruit maturation, and agricultural factors. The technology used for juice processing may affect antioxidant capacity of pomegranate juice [7]. In this regard, the antioxidant activity of commercial pomegranate juice was 50\% higher 
TABLE 3: Evaluation of total polyphenol content (mg gallic acid equivalents/L) and antioxidant activities based on ABTS (mg ascorbic $\mathrm{acid} / 100 \mathrm{~mL})$, FRAP $\left(\mathrm{mmol} \mathrm{Fe} \mathrm{e}^{2+} / 100 \mathrm{~mL}\right)$, and DPPH (\%AA) in pomegranate juices.

\begin{tabular}{|c|c|c|c|c|}
\hline Pomegranate juice & Total phenolic (mg/L) & ABTS $(\mathrm{mg} / 100 \mathrm{~mL})$ & FRAP $(\mathrm{mmol} / 100 \mathrm{~mL})$ & DPPH (\%AA) \\
\hline VKTA & $360.5 \pm 14.5^{\mathrm{e} *}$ & $222.3 \pm 6.8^{f}$ & $15.8 \pm 0.4^{\mathrm{de}}$ & $25.6 \pm 1.7^{\mathrm{de}}$ \\
\hline GSYA & $590.3 \pm 25.1^{\mathrm{cd}}$ & $407.0 \pm 17.0^{\mathrm{d}}$ & $20.2 \pm 1.3^{\mathrm{bc}}$ & $35.5 \pm 1.3^{\mathrm{bc}}$ \\
\hline MYA & $906.0 \pm 12.7^{\mathrm{b}}$ & $551.7 \pm 16.6^{\mathrm{b}}$ & $23.6 \pm 1.8^{\mathrm{ab}}$ & $37.3 \pm 2.1^{\mathrm{bc}}$ \\
\hline MTKA & $827.0 \pm 29.7^{\mathrm{b}}$ & $497.4 \pm 13.6^{\mathrm{c}}$ & $22.9 \pm 0.3^{\mathrm{ab}}$ & $39.8 \pm 2.4^{\mathrm{ab}}$ \\
\hline JPGRTA & $1266.8 \pm 44.2^{\mathrm{a}}$ & $620.0 \pm 19.8^{\mathrm{a}}$ & $25.9 \pm 1.3^{\mathrm{a}}$ & $46.8 \pm 2.6^{\mathrm{a}}$ \\
\hline TMLA & $562.0 \pm 9.2^{\mathrm{d}}$ & $312.4 \pm 6.5^{\mathrm{e}}$ & $17.1 \pm 0.9^{\mathrm{cd}}$ & $31.2 \pm 0.1^{\mathrm{cd}}$ \\
\hline ATSA & $590.3 \pm 20.9^{c d}$ & $311.3 \pm 7.5^{\mathrm{e}}$ & $20.7 \pm 0.3^{\mathrm{bc}}$ & $35.1 \pm 1.8^{\mathrm{bc}}$ \\
\hline SRABA & $671.3 \pm 21.6^{c}$ & $398.2 \pm 9.6^{\mathrm{d}}$ & $19.6 \pm 1.7^{\mathrm{cd}}$ & $34.7 \pm 1.8^{\mathrm{bc}}$ \\
\hline PSYA & $220.0 \pm 1.4^{\mathrm{f}}$ & $117.1 \pm 3.3^{\mathrm{g}}$ & $13.6 \pm 0.1^{\mathrm{e}}$ & $18.8 \pm 1.4^{\mathrm{e}}$ \\
\hline MPSA & $536.3 \pm 17.3^{\mathrm{d}}$ & $364.2 \pm 4.7^{\mathrm{d}}$ & $16.6 \pm 1.3^{\mathrm{cd}}$ & $28.1 \pm 1.7^{\mathrm{de}}$ \\
\hline VKTW & $1270.8 \pm 29.5^{\mathrm{f}}$ & $614.7 \pm 23.6^{\mathrm{e}}$ & $23.3 \pm 0.7^{\mathrm{d}}$ & $45.1 \pm 2.8^{\mathrm{d}}$ \\
\hline GSYW & $2931.5 \pm 82.5^{\mathrm{a}}$ & $1640.1 \pm 66.3^{\mathrm{a}}$ & $59.9 \pm 4.3^{\mathrm{a}}$ & $71.3 \pm 4.9^{\mathrm{a}}$ \\
\hline MYW & $1854.7 \pm 32.3^{\mathrm{de}}$ & $1247.0 \pm 24.0^{c}$ & $40.7 \pm 2.1^{\mathrm{b}}$ & $62.3 \pm 4.7^{b c}$ \\
\hline MTKW & $1698.8 \pm 62.0^{\mathrm{e}}$ & $1221.2 \pm 14.9^{c}$ & $37.0 \pm 1.6^{\mathrm{b}}$ & $53.6 \pm 3.2^{c}$ \\
\hline JPGRTW & $2568.0 \pm 52.9^{\mathrm{b}}$ & $1431.7 \pm 44.2^{\mathrm{b}}$ & $54.8 \pm 3.0^{\mathrm{a}}$ & $65.6 \pm 5.0^{\mathrm{bc}}$ \\
\hline TMLW & $1955.1 \pm 69.4^{\mathrm{cd}}$ & $1222.5 \pm 19.0^{c}$ & $35.7 \pm 2.1^{b c}$ & $60.5 \pm 5.0^{c}$ \\
\hline ATSW & $2136.1 \pm 48.0^{c}$ & $1139.8 \pm 13.1^{\mathrm{cd}}$ & $33.8 \pm 2.9^{c}$ & $63.5 \pm 2.0^{\mathrm{bc}}$ \\
\hline SRABW & $1834.3 \pm 52.8^{\mathrm{de}}$ & $997.0 \pm 18.4^{\mathrm{d}}$ & $31.6 \pm 1.8^{\mathrm{c}}$ & $58.0 \pm 3.3^{c}$ \\
\hline PSYW & $942.9 \pm 43.4^{\mathrm{g}}$ & $496.2 \pm 13.0^{\mathrm{f}}$ & $22.6 \pm 0.6^{\mathrm{d}}$ & $36.6 \pm 2.3^{\mathrm{e}}$ \\
\hline MPSW & $1954.1 \pm 27.1^{\mathrm{cd}}$ & $1249.2 \pm 26.5^{c}$ & $30.6 \pm 1.8^{c}$ & $55.0 \pm 1.8^{\mathrm{c}}$ \\
\hline
\end{tabular}

${ }^{*}$ Different letters in the same column present significant difference at $p<0.05$.

than juices from pomegranate arils, due to the entrance of phenolic compounds from the peel into juices [1]. Also, Tzulker et al. [11] reported that antioxidant activity of whole fruit homogenates is about 20 -fold higher than that found in the arils. These results indicated that hydrolysable tannins including punicalagin were the main compounds influencing the antioxidant capacity of pomegranate juices $[1,11]$. This compound shows a high antioxidant activity due to the 16 phenolic hydroxyl groups in the molecule [1].

Moreover, a good correlation was observed between total phenolic content and ABTS and FRAP methods in all pomegranate juices $(>0.90)$. Correlations between total phenolic and DPPH in juices of arils and whole pomegranate were 0.92 and 0.86 , respectively. However, Pearson correlation showed that the correlation of three measurement antioxidant methods is affected by pomegranate cultivars, as mentioned in previous research $[7,11]$. In this regard, Tzulker et al. [11] reported that the antioxidant activity (FRAP and $\mathrm{DPPH}$ methods) of juices from whole pomegranate homogenate and arils alone significantly correlated with the polyphenol level, but it did not correlate with anthocyanins level. The highest correlation value was obtained between antioxidant activity and punicalagin in the whole pomegranate homogenate. The FRAP method compared to DPPH method gave the highest correlation with total polyphenol content.

\section{Conclusion}

The individual and total phenolic contents of all studied cultivars were significantly different. In this study, among nine major and minor anthocyanins, the cyanidinpentoside, cyanidin-pentoside-hexoside, and delphinidinpentoside were identified for the first time in Iranian pomegranate cultivars. The phenolic contents and antioxidant activity of whole pomegranate juice were significantly higher than those of juices from pomegranate arils from the same cultivar, due to the entrance of phenolic compounds from the rind parts of pomegranate to the juices. The variation between the cultivars is very wide; therefore, the contents of ellagitannins and anthocyanins in the juice from whole pomegranates of the low-polyphenol cultivars (as MY) are lower than those in the juices made from arils only of the high-polyphenol cultivars (as JPGRT). In other words, the cultivar has a higher influence on the phenolic content of the juice than the extraction method.

\section{Conflict of Interests}

The authors declare that there is no conflict of interests regarding the publication of this paper.

\section{Acknowledgment}

The authors would like to acknowledge Dr. Vazifeshenas for his assistance in afforded pomegranate cultivars.

\section{References}

[1] M. I. Gil, F. A. Tomas-Barberan, B. Hess-Pierce, D. M. Holcroft, and A. A. Kader, "Antioxidant activity of pomegranate juice 
and its relationship with phenolic composition and processing," Journal of Agricultural and Food Chemistry, vol. 48, no. 10, pp. 4581-4589, 2000.

[2] U. A. Fischer, R. Carle, and D. R. Kammerer, "Identification and quantification of phenolic compounds from pomegranate (Punica granatum L.) peel, mesocarp, aril and differently produced juices by HPLC-DAD-ESI/MS ${ }^{n}$, Food Chemistry, vol. 127, no. 2, pp. 807-821, 2011.

[3] Anonymous, Iran Statistical Year Book 2013, 2013, http://eamar .sci.org.ir/index_e.aspx.

[4] P. Mena, C. García-Viguera, J. Navarro-Rico et al., "Phytochemical characterisation for industrial use of pomegranate (Punica granatum L.) cultivars grown in Spain," Journal of the Science of Food and Agriculture, vol. 91, no. 10, pp. 1893-1906, 2011.

[5] G. Mousavinejad, Z. Emam-Djomeh, K. Rezaei, and M. H. H. Khodaparast, "Identification and quantification of phenolic compounds and their effects on antioxidant activity in pomegranate juices of eight Iranian cultivars," Food Chemistry, vol. 115, no. 4, pp. 1274-1278, 2009.

[6] O. A. Fawole, U. L. Opara, and K. I. Theron, "Chemical and phytochemical properties and antioxidant activities of three pomegranate cultivars grown in South Africa," Food and Bioprocess Technology, vol. 5, no. 7, pp. 2934-2940, 2012.

[7] M. Çam, Y. Hışıll, and G. Durmaz, "Classification of eight pomegranate juices based on antioxidant capacity measured by four methods," Food Chemistry, vol. 112, no. 3, pp. 721-726, 2009.

[8] F. Tezcan, M. Gültekin-Özgüven, T. Diken, B. Özçelik, and F. B. Erim, "Antioxidant activity and total phenolic, organic acid and sugar content in commercial pomegranate juices," Food Chemistry, vol. 115, no. 3, pp. 873-877, 2009.

[9] A. Tehranifar, M. Zarei, Z. Nemati, B. Esfandiyari, and M. R. Vazifeshenas, "Investigation of physico-chemical properties and antioxidant activity of twenty Iranian pomegranate (Punica granatum L.) cultivars," Scientia Horticulturae, vol. 126, no. 2, pp. 180-185, 2010.

[10] M. Ozgen, C. Durgaç, S. Serçe, and C. Kaya, "Chemical and antioxidant properties of pomegranate cultivars grown in the Mediterranean region of Turkey," Food Chemistry, vol. 111, no. 3, pp. 703-706, 2008.

[11] R. Tzulker, I. Glazer, I. Bar-Ilan, D. Holland, M. Aviram, and R. Amir, "Antioxidant activity, polyphenol content, and related compounds in different fruit juices and homogenates prepared from 29 different pomegranate accessions," Journal of Agricultural and Food Chemistry, vol. 55, no. 23, pp. 9559-9570, 2007.

[12] H. Alighourchi, M. Barzegar, and S. Abbasi, "Anthocyanins characterization of 15 Iranian pomegranate (Punica granatum L.) varieties and their variation after cold storage and pasteurization," European Food Research and Technology, vol. 227, no. 3, pp. 881-887, 2008.

[13] S. Chrubasik-Hausmann, C. Vlachojannis, and B. Zimmermann, "Pomegranate juice and prostate cancer: importance of the characterisation of the active principle," Phytotherapy Research, vol. 28, no. 11, pp. 1676-1678, 2014.

[14] P. Mena, L. Calani, C. Dall'Asta et al., "Rapid and comprehensive evaluation of (Poly)phenolic compounds in pomegranate (Punica granatum L.) Juice by UHPLC-MSn," Molecules, vol. 17, no. 12, pp. 14821-14840, 2012.

[15] M. M. Feuereisen, J. Hoppe, B. F. Zimmermann, F. Weber, N. Schulze-Kaysers, and A. Schieber, "Characterization of phenolic compounds in Brazilian pepper (Schinus terebinthifolius Raddi) exocarp," Journal of Agricultural and Food Chemistry, vol. 62, no. 26, pp. 6219-6226, 2014.

[16] S. Vegara, N. Martí, J. Lorente et al., "Chemical guide parameters for Punica granatum cv. 'Mollar' fruit juices processed at industrial scale," Food Chemistry, vol. 147, pp. 203-208, 2014.

[17] G. Miguel, S. Dandlen, D. Antunes, A. Neves, and D. Martins, "The effect of two methods of pomegranate (Punica granatum L) juice extraction on quality during storage at $4^{\circ} \mathrm{C}$," Journal of Biomedicine and Biotechnology, vol. 2004, no. 5, pp. 332-337, 2004.

[18] A. Pérez-Vicente, P. Serrano, P. Abellán, and C. García-Viguera, "Influence of packaging material on pomegranate juice colour and bioactive compounds, during storage," Journal of the Science of Food and Agriculture, vol. 84, no. 7, pp. 639-644, 2004.

[19] N. Martí, A. Pérez-Vicente, and C. García-Viguera, "Influence of storage temperature and ascorbic acid addition on pomegranate juice," Journal of the Science of Food and Agriculture, vol. 82, no. 2, pp. 217-221, 2002.

[20] Ç. U. Pala and A. K. Toklucu, "Effect of UV-C light on anthocyanin content and other quality parameters of pomegranate juice," Journal of Food Composition and Analysis, vol. 24, no. 6, pp. 790-795, 2011.

[21] F. Shahidi and M. Naczk, Phenolics in Food and Nutraceuticals, CRC Press, Boca Raton, Fla, USA, 2004.

[22] A. Patras, N. P. Brunton, S. Da Pieve, and F. Butler, "Impact of high pressure processing on total antioxidant activity, phenolic, ascorbic acid, anthocyanin content and colour of strawberry and blackberry purées," Innovative Food Science \& Emerging Technologies, vol. 10, no. 3, pp. 308-313, 2009. 

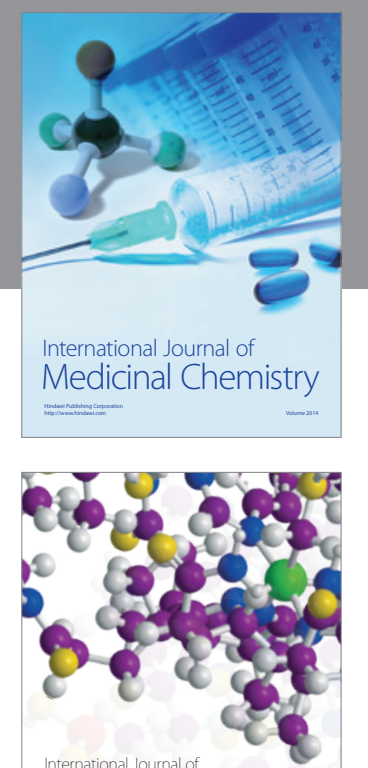

\section{Carbohydrate} Chemistry

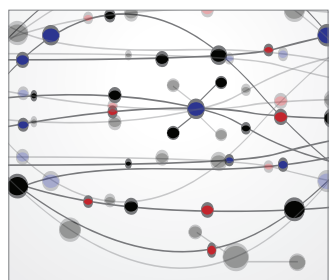

The Scientific World Journal
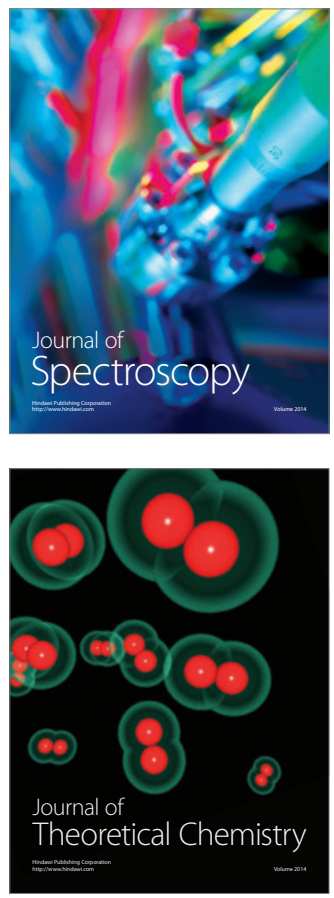
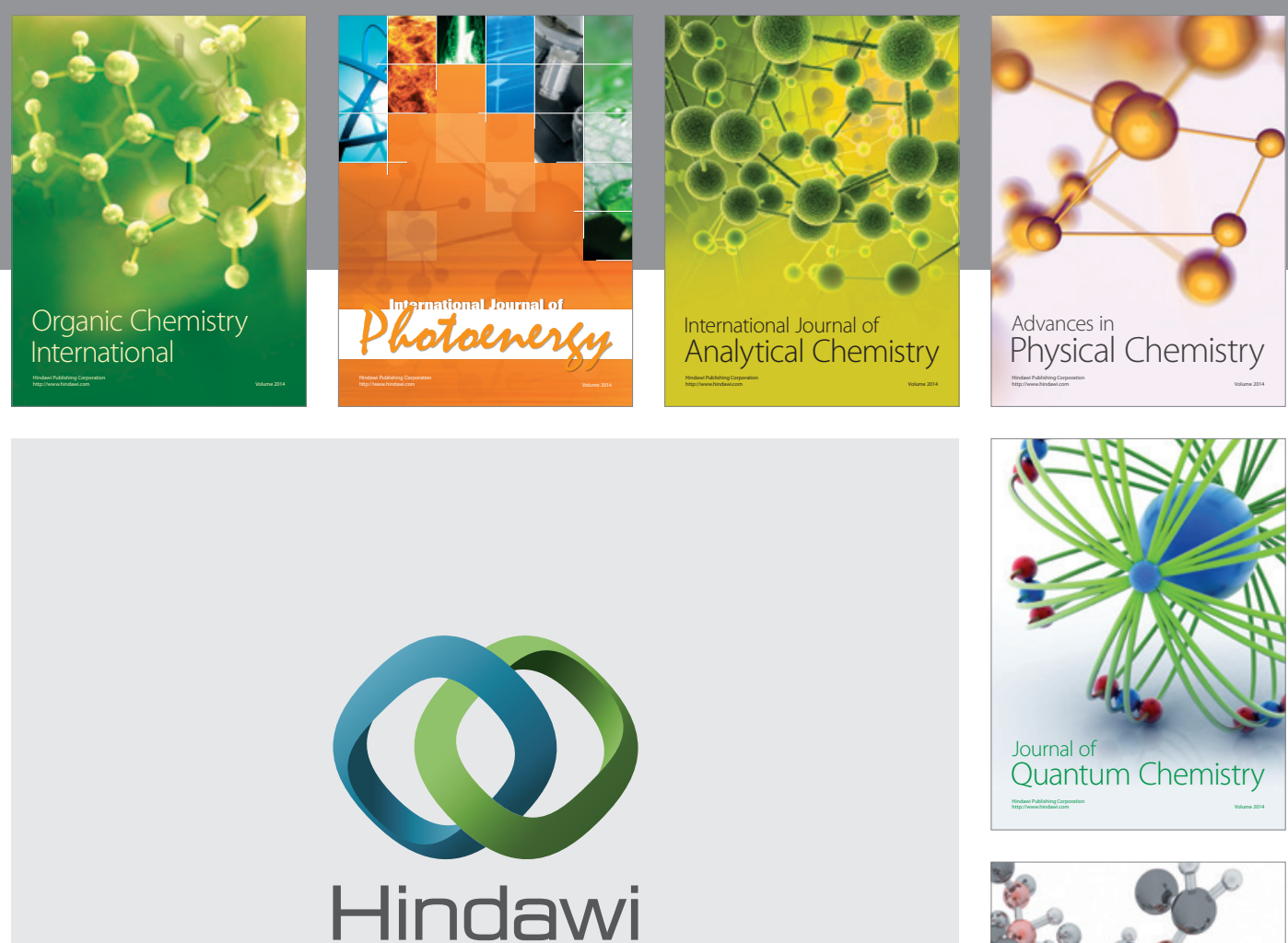

Submit your manuscripts at

http://www.hindawi.com

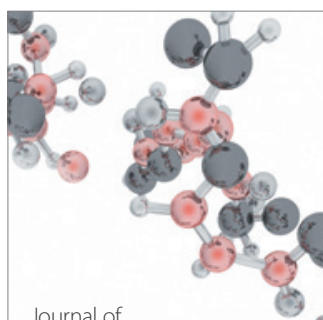

Analytical Methods

in Chemistry

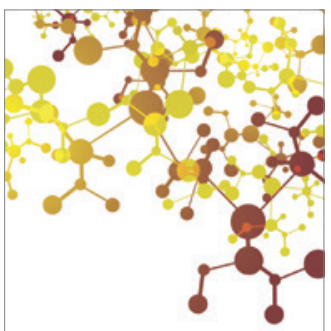

Journal of

Applied Chemistry

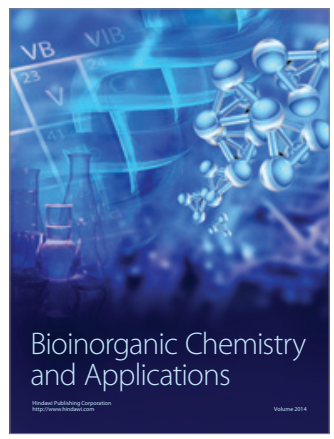

Inorganic Chemistry
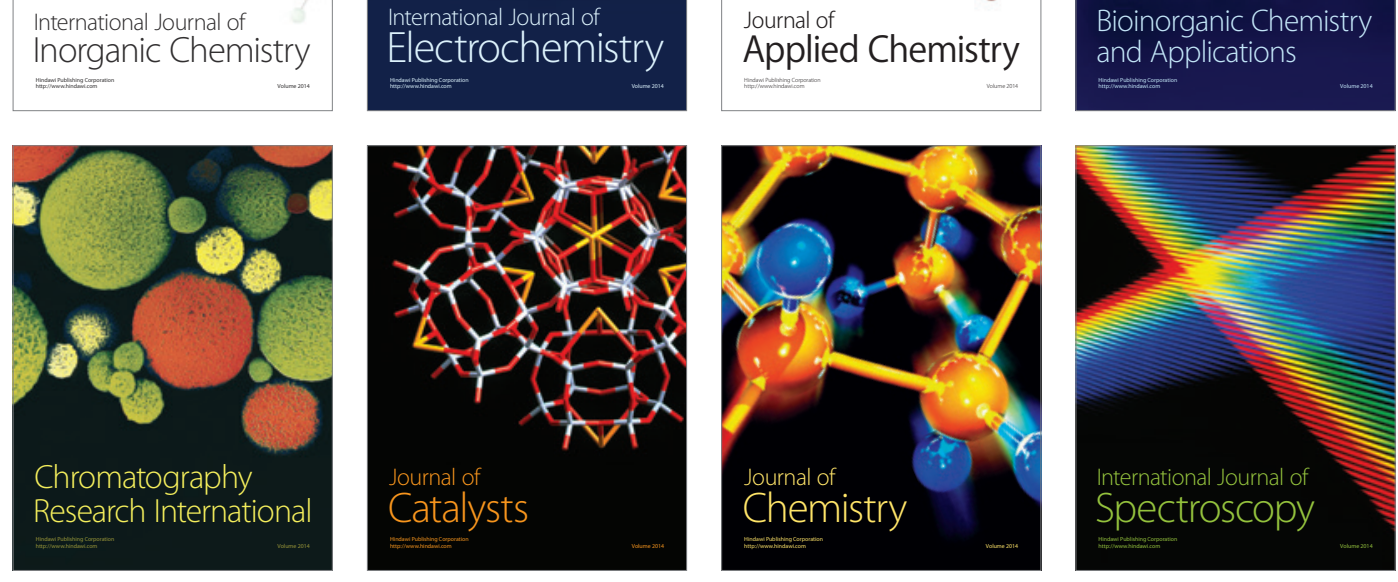